\title{
STANDARD MODEL MEASUREMENTS WITH ATLAS (+CMS) $\operatorname{DETECTOR}(\mathrm{S})$
}

\author{
SHU LI, ON BEHALF OF THE ATLAS COLLABORATION \\ Department of Physics, Duke University, Physics Bldg., Science Dr., Box 90305 \\ Durham, NC 27708, United States of America \\ shuli@phy.duke.edu
}

Received 31/08/2015

Revised $\mathrm{xx} / \mathrm{xx} / 2015$

Published $\mathrm{xx} / \mathrm{xx} / 2015$

\begin{abstract}
We present a broad range of Standard Model measurements performed in protonproton collisions at the LHC on a variety of topics $W / Z+$ jets production and ratios, the electroweak multi-boson production, $W / Z$ heavy flavor decay production, jet production, photon physics, the Standard Model soft QCD and diffractive physics. The latest public results from ATLAS experiment are obtained at the center-of-mass energy of both 7 $\mathrm{TeV}$ and $8 \mathrm{TeV}$, which covers total cross sections, fiducial cross sections, differential cross sections wherever available and comparison with Leading-Order/Next-to-LeadingOrder/Next-to-Next-to-Leading-Order theory predictions. These measurements provide important tests for perturbative QCD, electroweak multi-boson interactions, Electroweak Symmetry Breaking over a broad kinematic range and constraints for PDFs. Modelindependent new physics searches are also performed by probing anomalous gauge bosons couplings using effective Lagrangian or effective field theory formalism.
\end{abstract}

Keywords: Standard Model; Measurement; Electroweak; QCD; Photon; Jet; Boson; Lepton; Neutrino; High Energy; ATLAS; CMS; LHC.

PACS numbers:

\section{SM $W / Z$ Physics}

We present the measurements of Standard Model (SM) Vector Boson $(W / Z)+$ Jets involving the following physics topics according to the latest publications and conference results by ATLAS: the $W+$ jets differential cross section, the ratio of the cross section of $W$ and $Z$ boson with jets, the distribution of $Z / \gamma^{*}$ boson $p_{\mathrm{T}}$ (transverse momentum), the high $p_{\mathrm{T}}$ single boson jet production cross section. All measurements are done with $4.6 \mathrm{fb}^{-1} 7 \mathrm{TeV}$ data taken in 2011 by ATLAS ${ }^{1}$.

The $W+$ jets differential cross section is measured using fully leptonic decay final states $\left(e^{ \pm}\right.$or $\left.\mu^{ \pm}\right) .{ }^{5}$ The full $7 \mathrm{TeV}$ collision data taken in the year of 2011 allows for a precise measurement of the $W+$ jets cross section for the first time up to seven

This is an Open Access article published by World Scientific Publishing Company. It is distributed under the terms of the Creative Commons Attribution 3.0 (CC-BY) License. Further distribution of this work is permitted, provided the original work is properly cited. 
jets and an exploration of a large kinematic range, including jet production up to a transverse momentum of $1 \mathrm{TeV}$. The comparison are made with numerous QCD predictions: next-to-leading-order perturbative calculations, resummation calculations and Monte Carlo generators. Fixed-order predictions (BlackHat+SHERPA) in general provide good description of data but still have mis-modeling of some variables such as $H_{T}$ or $S_{T}$ in dijet dominant regions with $W$ boson emitted from one of the quarks.

The Rjet variable (ratio of the cross section of $W$ and $Z$ boson with jets) provides more precise test of perturbative QCD (pQCD) than individual $\mathrm{V}+$ jets measurements because it cancels greatly the experimental uncertainties and non-pQCD effects in the ratio. The measurement is made for the first time up to four jets ${ }^{6}$, which helps to validate the latest state-of-the-art Next-to-Leading-Order (NLO) pQCD calculations in most regions (particularly BlackHat+SHERPA). The successful measurement, which in good agreement with NLO theory predictions as shown in Figure 1, inspires a further tuning of the MC generator to improve the description of the production of $\mathrm{V}+$ jets.

The measurement of $Z / \gamma^{*} p_{\mathrm{T}}$ spectrum is performed in both $Z / \gamma^{*} \rightarrow e^{+} e^{-}$ and $Z / \gamma^{*} \rightarrow \mu^{+} \mu^{-}$channels. ${ }^{7}$ The normalized differential cross sections in $p_{\mathrm{T}}^{Z}$ are measured up to $800 \mathrm{GeV}$ in three rapidity ranges: $0 \leq y_{Z}<1.1,1.1 \leq y_{Z}<2$, $2 \leq y_{Z}<2.4$, which are in comparison with $\mathrm{pQCD}$ and resummed predictions using various MC generators. The measured $p_{\mathrm{T}}^{Z}$ and and $\phi^{*}$ distributions are used to tune the Pythia8 and Powheg + Pythia8 generators.

The single boson jet measurement is motivated in boosted region where close-by outgoing quarks from high $p_{\mathrm{T}}$ bosons can merge into one fat jet after hadronization and clustering. By reconstructing $W$ and $Z$ boson hadron decay products in one single jet with boson mass constraint and identification methodology using jet energy cluster substructure information in the jet centre-of-mass frame, the large QCD multi-jet background can be significantly suppressed. The boson jet $p_{\mathrm{T}}$ is measured up to $320 \mathrm{GeV}$ and $|\eta|$ (pseudo-rapidity) up to 1.9. The cross section of single boson jet production decayed from $W$ and $Z$ is measured to be: $8.5 \pm 0.8$ (stat.) \pm 1.5 (syst.) pb which is within $2 \sigma$ of the NLO QCD prediction of $\sigma_{W+Z}=5.1 \pm 0.5 \mathrm{pb} .{ }^{8}$

A full summary of all the currently published ATLAS ${ }^{1}$ and CMS ${ }^{2,3}$ single vector boson $(W / Z)+$ jets measurement results and comparison with theory predictions is shown in Figure 2 and 3.

\section{SM Electroweak Physics}

We present the latest SM electroweak measurements of ATLAS with main focus on Multi-Boson production. The precisely measured multi-boson production total, fiducial and differential cross sections scrutinise the SM electroweak sector with a hadron collider at the $\mathrm{TeV}$ scale and provide a stringent test of NLO electroweak and NLO/Next-to-Next-to-Leading-Order (NNLO) QCD calculations. It also inspires the BSM (Beyond SM) new physics searches by means of thoroughly under- 
standing the irreducible multi-boson backgrounds from SM electroweak production. The follow topics are to be covered: first evidence of Vector Boson Scattering (VBS) production at $8 \mathrm{TeV}$ in same-electric-charge $W^{ \pm} W^{ \pm}$channel, first evidence of triboson production at $8 \mathrm{TeV}$ in $W^{ \pm} \gamma \gamma$ channel, $W^{ \pm} V(j j)$ production cross section measurements at $7 \mathrm{TeV}, 7 / 8 \mathrm{TeV} Z \rightarrow 4 l$ cross section and branching ratio (BR) measurements, measurement of the forward-backward asymmetry $\left(A_{F B}\right)$ of lepton pair production and Weinberg angle extraction at $7 \mathrm{TeV}$, simultaneous measurement of the $t \bar{t}, W^{ \pm} W^{ \pm}$and $Z \rightarrow \tau^{ \pm} \tau^{ \pm}$production cross section at $7 \mathrm{TeV}$. Model independent new physics searches are made by probing the anomalous gauge bosons coupling vertices.

The evidence of VBS production at $8 \mathrm{TeV}$ is claimed by ATLAS for the first time and measured in same-electric-charge $W^{ \pm} W^{ \pm}$fully leptonic final states $(e / \mu)$ with $20.3 \mathrm{fb}^{-1} 8 \mathrm{TeV}$ data. ${ }^{9}$ The representative Feynman diagrams are shown in Figure 4 for electroweak signal (including both VBS and non-VBS production) and in Figure 5 for irreducible QCD multijet background. The signal is extracted in a VBS search region: $\Delta y(j j)>2.4, M(j j)>500 \mathrm{GeV}$. The fiducial cross section in this region is measured to be in agreement with SM predictions. The evidence of

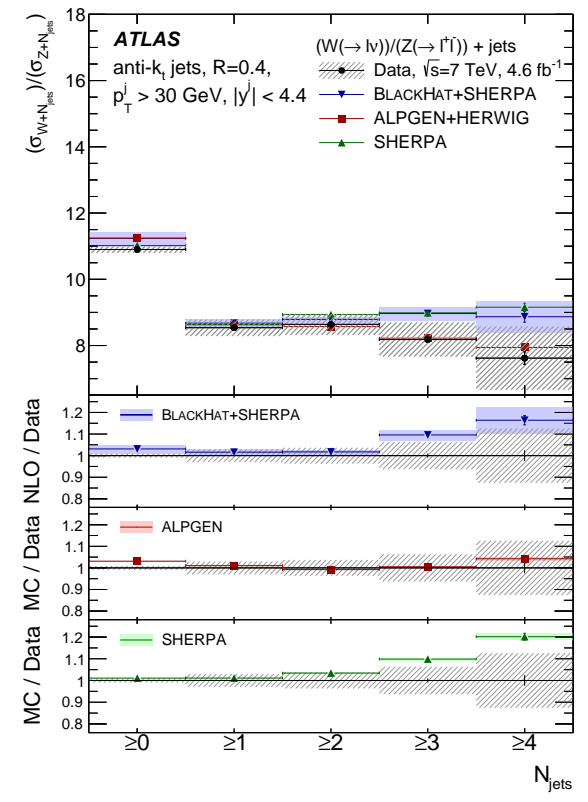

(a)

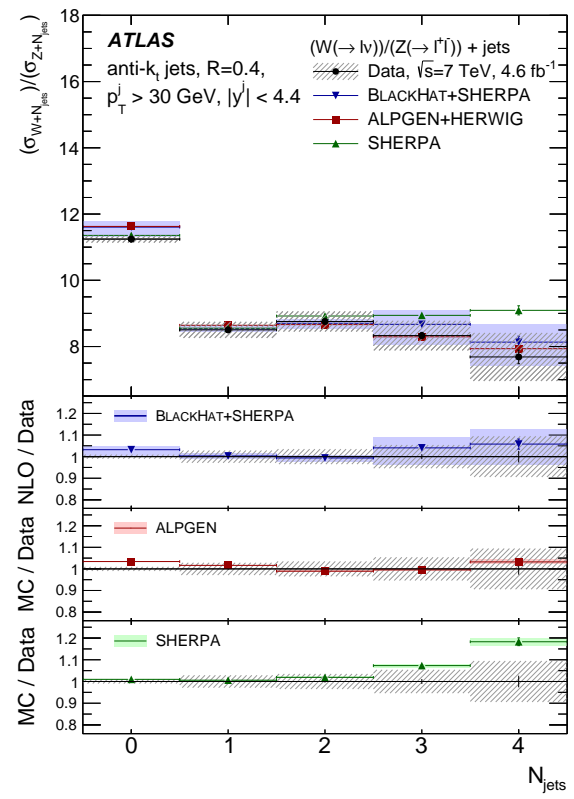

(b)

Fig. 1. Rjets as a function of exclusive jet multiplicity (left) and inclusive jet multiplicity (right) with electron and muon channels combined. A thorough comparison across all jet multiplicity range with BlackHat+SHERPA NLO calculation and the ALPGEN and SHERPA generators are shown at the bottom of each plot which show good agreement with theory prediction in general except for SHERPA in high jet multiplicity bins. ${ }^{6}$ 


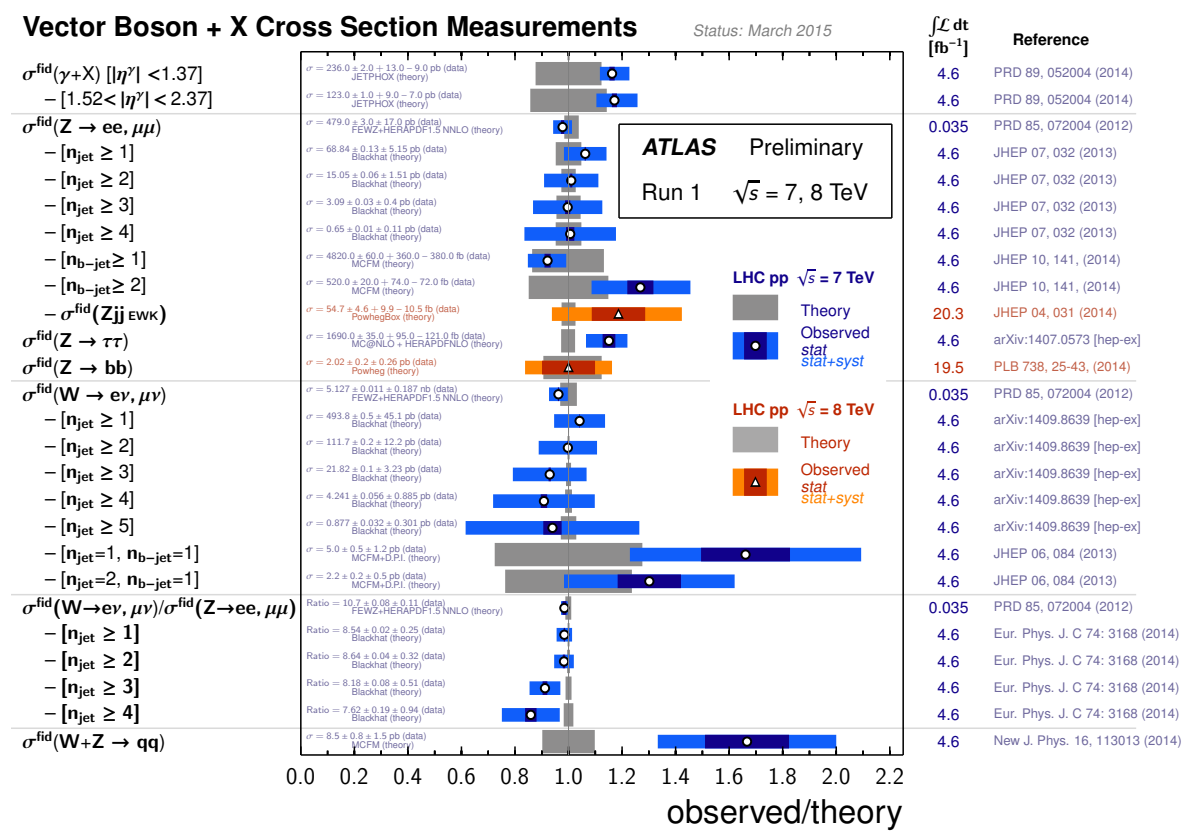

Fig. 2. Summary of current ATLAS single vector boson $(W / Z)+$ jets measurement results. ${ }^{4}$

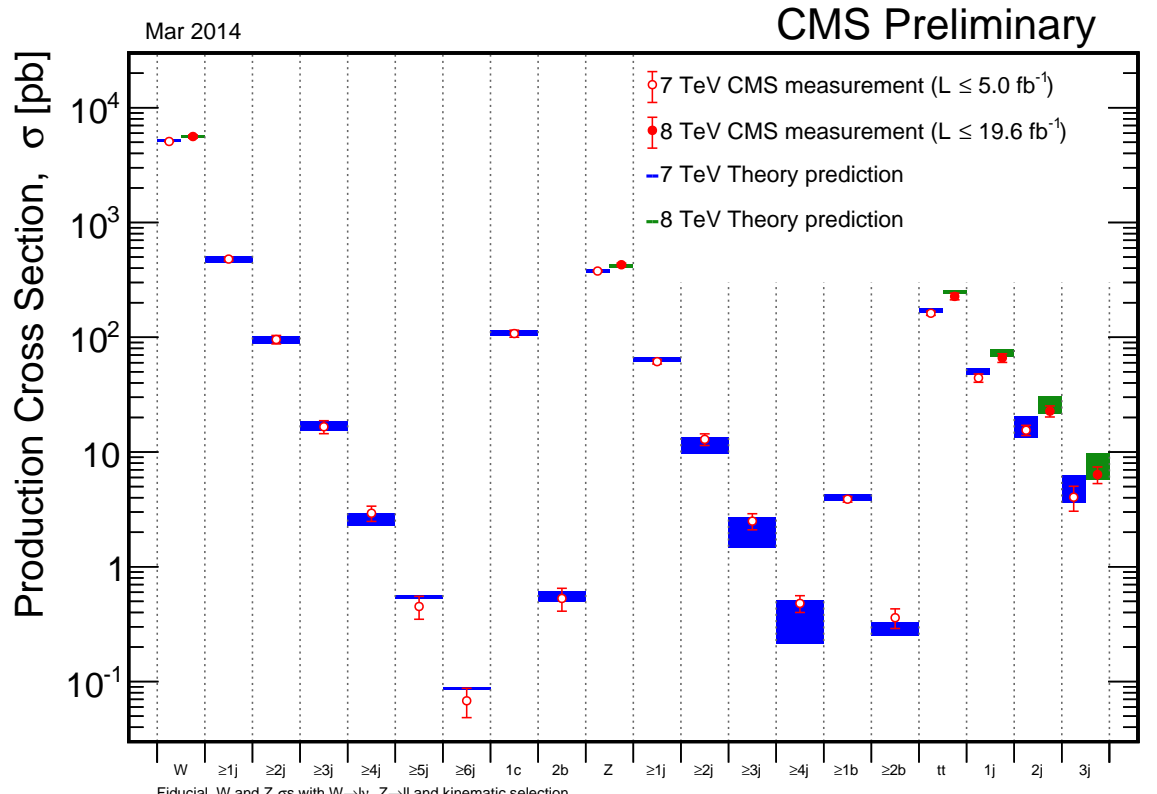

Fig. 3. Summary of current CMS single vector boson $(W / Z)+$ jets measurement results. ${ }^{3}$ 
$W^{ \pm} W^{ \pm}$and electroweak-only $W^{ \pm} W^{ \pm}$is established with a significance of 4.5 and 3.6 standard deviations, respectively. Left plot on Figure 6 shows the M(jj) distribution passing inclusive selection with a cut on $M(j j)>500 \mathrm{GeV}$ for background rejection. Right plot on Figure 6 presents the first 2-D anomalous quartic gauge coupling (aQGC) limits of high dimension operators $\alpha_{4} / \alpha_{5}$ using measured fiducial cross-section. The k-matrix unitarization treatment is adopted to avoid unitarity violation. ${ }^{9}$

The evidence of triboson production at $8 \mathrm{TeV}$ is concluded in fully leptonic final states of $W^{ \pm} \gamma \gamma$ production with two isolated photons by ATLAS with $20.3 \mathrm{fb}^{-1} 8$ $\mathrm{TeV}$ data. ${ }^{10}$ Fiducial cross sections are measured in both inclusive and exclusive jet channels. In comparison with NLO MCFM calculation, exclusive jet channel shows good agreement while inclusive cross section is higher by 1.9 standard deviations. The first aQGC limits parameterized with effective field theory (EFT) dimension eight operators are unitarized with dipole form factor $(\mathrm{FF})$ treatment. The exclusive jet channel with a further cut on $M(\gamma \gamma)>300 \mathrm{GeV}$ is used for the limit setting. The aQGC limits together with the $M(\gamma \gamma)$ spectrum are shown in Figure 7.

The combined production cross section of $W W+W Z$ is measured in semileptonic final states with one of the $W / Z$ decaying into hadrons using $4.6 \mathrm{fb}^{-1} 7 \mathrm{TeV}$ data. ${ }^{11}$ The fiducial cross section $68 \pm 7$ (stat.) \pm 19 (syst.) pb is measured with a $3.4 \sigma$ evidence and consistent with SM prediction $61.1 \pm 2.2 \mathrm{pb}$. The dijet transverse momentum spectrum is used to set limits on anomalous triple gauge coupling (aTGC) vertices parameterized with EFT high dimension operators.

The cross sections and BR of $Z \rightarrow 4 l$ production from $Z$ resonance are measured with both $7 \mathrm{TeV}$ and $8 \mathrm{TeV}$ full data. ${ }^{12}$ The backgrounds consists of non-resonant $4 l$ and other dibosons. The measured cross section at $7 \mathrm{TeV}(76 \pm 18$ (stat.) \pm 4 (syst.) \pm 1.4 (lumi.) fb and $107 \pm 9$ (stat.) \pm 4 (syst.) \pm 3.0 (lumi.) fb at 8 $\mathrm{TeV}$ which are consistent with the SM predictions of $90.0 \pm 2.1 \mathrm{fb}$ and $104.8 \pm$ $2.5 \mathrm{fb}$, respectively. By normalizing to the measured $Z \rightarrow \mu^{+} \mu^{-}$production, we measure the BR of $Z \rightarrow 4 l$ to be $(3.20 \pm 0.25$ (stat.) \pm 0.13 (syst.) $) \times 10^{-6}$ in good agreement with the SM prediction of $(3.33 \pm 0.01) \times 10^{-6}$.

The $A_{F B}$ is measured in the process of $p p \rightarrow Z / \gamma^{*} \rightarrow l^{+} l^{-}(l$ being $e$ or $\mu)$ with a $4.8 \mathrm{fb}^{-1} 7 \mathrm{TeV}$ data of $2011 .{ }^{13}$ By combining electron and muon channels, the effective mixing angle is measured to be $\sin ^{2} \theta_{\text {eff }}^{\text {lept }}=0.2308 \pm 0.0005$ (stat.) \pm 0.0006 (syst.) \pm 0.0009 (PDF), which agrees with the current Particle Data Group (PDG) fitted world average value. The results are in good agreement with other experiments given the current precision.

The fiducial cross sections of $t \bar{t}, W^{+} W^{-}$and $Z \rightarrow \tau \tau$ are simultaneously measured in common final states consisting of opposite charge high $p_{\mathrm{T}} e \mu$ pairs with $4.6 \mathrm{fb}^{-1} 7 \mathrm{TeV}$ data. ${ }^{14}$ The $H \rightarrow W^{+} W^{-}$is also accounted for as part of the small contribution for $W^{+} W^{-}$production. The simultaneous measurements give a broader test than individual measurements and probe the effects of PDF with correlations accounted for between these processes. An improved understanding of these 
simultaneously measured processes will help to improve the corresponding theory calculations and SM background understanding in related new physics searches.

The summary of all the currently published ATLAS ${ }^{1}$ and CMS ${ }^{2,3}$ Multi-Boson production cross section results divided by theory predictions is shown in Figure 8 and 9 .

\section{SM Jet and Photon Physics}

We present the latest ATLAS Jet and Photon differential measurements at $\sqrt{s}=7$ $\mathrm{TeV}$ covering the following topics: the measurement of inclusive-jet and three-jet

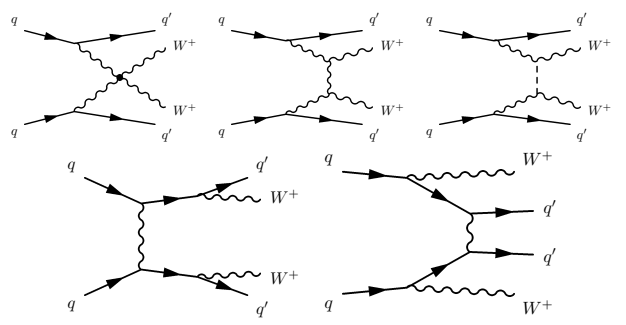

Fig. 4. The representative Feynman diagrams of electroweak-only same-electric-charge $W^{ \pm} W^{ \pm}$processes. The upper three diagrams show the VBS contributions while the bottom two show the non-VBS contributions. ${ }^{9}$

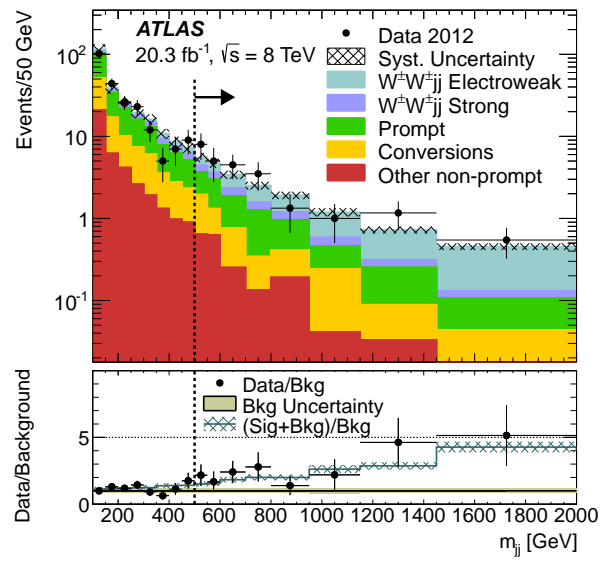

(a)

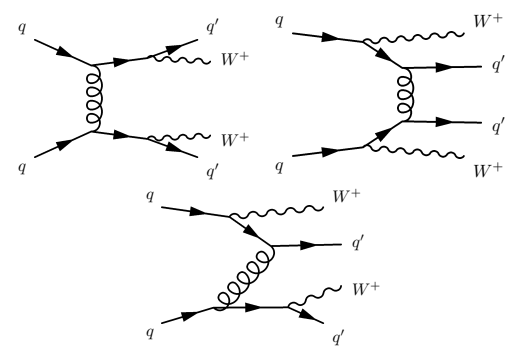

Fig. 5. The representative Feynman diagrams of same-electric-charge $W^{ \pm} W^{ \pm}$background processes with outgoing QCD partons. ${ }^{9}$

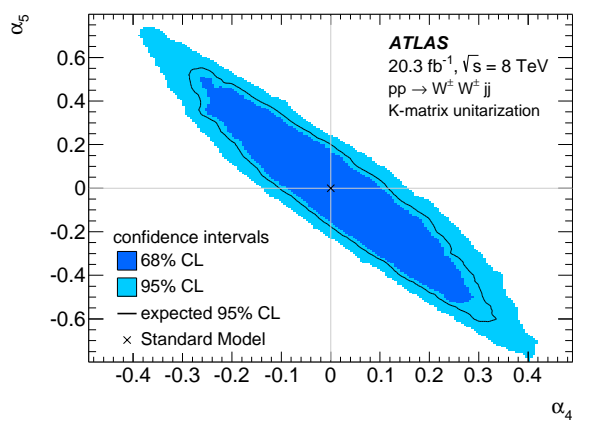

(b)

Fig. 6. Left: The same-electric-charge $W^{ \pm} W^{ \pm} \mathrm{M}(\mathrm{jj})$ distribution passing all fiducial cuts defined for the search region except $\mathrm{M}(\mathrm{jj})$ itself. Right: 2-D aQGC limits using measured fiducial crosssection with k-matrix unitarization. ${ }^{9}$ 
cross sections, the measurements of inclusive isolated prompt photon and photon pair cross sections.

Differential cross-sections of SM direct photon and photon pairs are measured in fiducial regions defined for a broad $E_{\mathrm{T}}$-range $(\tilde{\mathrm{T}} \mathrm{eV})$ and detector geometric coverage. The differential cross sections of SM inclusive isolated prompt photon are measured as a function of $E_{\mathrm{T}}(\gamma)([100,1000] \mathrm{GeV})$ and $|\eta(\gamma)|$ divided into barrel $(|\eta(\gamma)|<1.37)$ and endcap $(1.52<|\eta(\gamma)|<2.37) .{ }^{15}$ It agrees with the NLO predictions based on the CT10 and MSTW2008 PDF up to $1 \mathrm{TeV}$. The measured cross section of SM isolated photon pairs with $4.6 \mathrm{fb}^{-1} p p$ collision data at $7 \mathrm{TeV}$ within a fiducial region including both barrel and endcap for: $E_{\mathrm{T}}(\gamma)>22(25) \mathrm{GeV}, \Delta R(\gamma \gamma)>0.4 .{ }^{16}$ The comparisons with LO MC parton-shower (PS) models plus NLO pQCD calculations and NLO/NNLO generators are investigated. The measurements in general will provide a better understanding of the main backgrounds for many new physics searches with photons involved.

The SM inclusive jet and multi-jet production cross section measurements thoroughly test the pQCD calculations at NLO over a broad kinematic range (TeVscale). A variety of PDFs are considered for comparison to theory. Jets reconstructed with anti- $k_{t}$ algorithm and radius of both 0.4 and 0.6 are considered. The three-jet differential cross sections are measured as a function of three-jet mass for the first time up to $5 \mathrm{TeV}$ which are well described by pQCD at NLO on full $\left(M(j j j)\left|Y^{*}\right|\right)$ plane with a variety of PDFs. ${ }^{17}\left(Y^{*}\right.$ is defined from three jets's rapidities as $\left.Y^{*}=\left|y_{1}-y_{2}\right|+\left|y_{2}-y_{3}\right|+\left|y_{1}-y_{3}\right|\right)$ The inclusive jet production measurement considers $100 \mathrm{GeV}<p_{\mathrm{T}}<2 \mathrm{TeV}$ and a double-differential cross-section

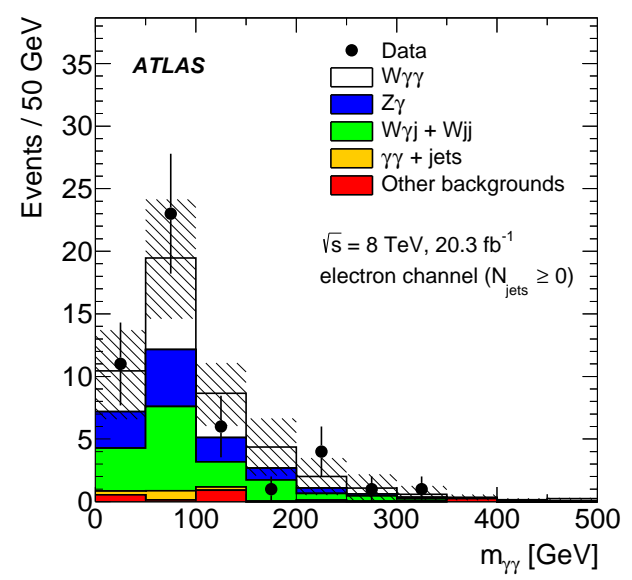

(a)

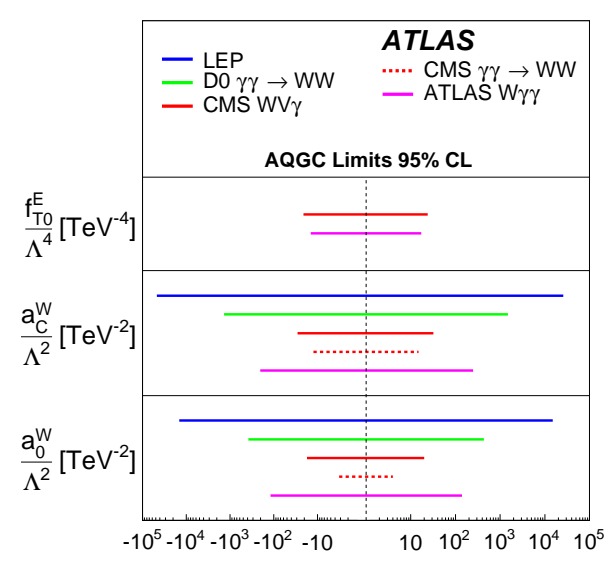

(b)

Fig. 7. Left: The $M(\gamma \gamma)$ distribution of data in comparison with predicted signal and estimated backgrounds of electron channel in inclusive jet channel. Right: aQGC limits using the measured high mass region in exclusive jet channel. ${ }^{10}$ 
as a function of $p_{\mathrm{T}}$ and $y .{ }^{18}$ The results are compared with NLO generators (Matrix Element + PS) and fixed-order NLO pQCD calculation accounting for both perturbative effect and electroweak effect. A summary of the current ATLAS measured inclusive jet cross sections are documented in Figure 10.

\section{SM Soft QCD and Diffractive Physics}

The following two major topics of Soft QCD and Diffractive Physics domain are highlighted in this report following up the latest publications of ATLAS: the exclusive dilepton production cross section and the Two-particle Bose-Einstein correlations (BEC) measurement.

The exclusive dilepton production cross sections are measured from $e e$ and $\mu \mu$ final states passing exclusive selection with the veto on additional charged-particle track activity. ${ }^{19}$ Signal is extracted by fitting the dilepton acoplanarity distributions to data. The measured cross section is consistent with theory prediction with correction of proton absorptive effects accounted. The $4.6 \mathrm{fb}^{-1} 7 \mathrm{TeV}$ data is used in the measurement. The acoplanarity distribution in $\mu \mu$ channel passing event selections and exclusivity requirements is shown in Figure 11 along with the comparison of

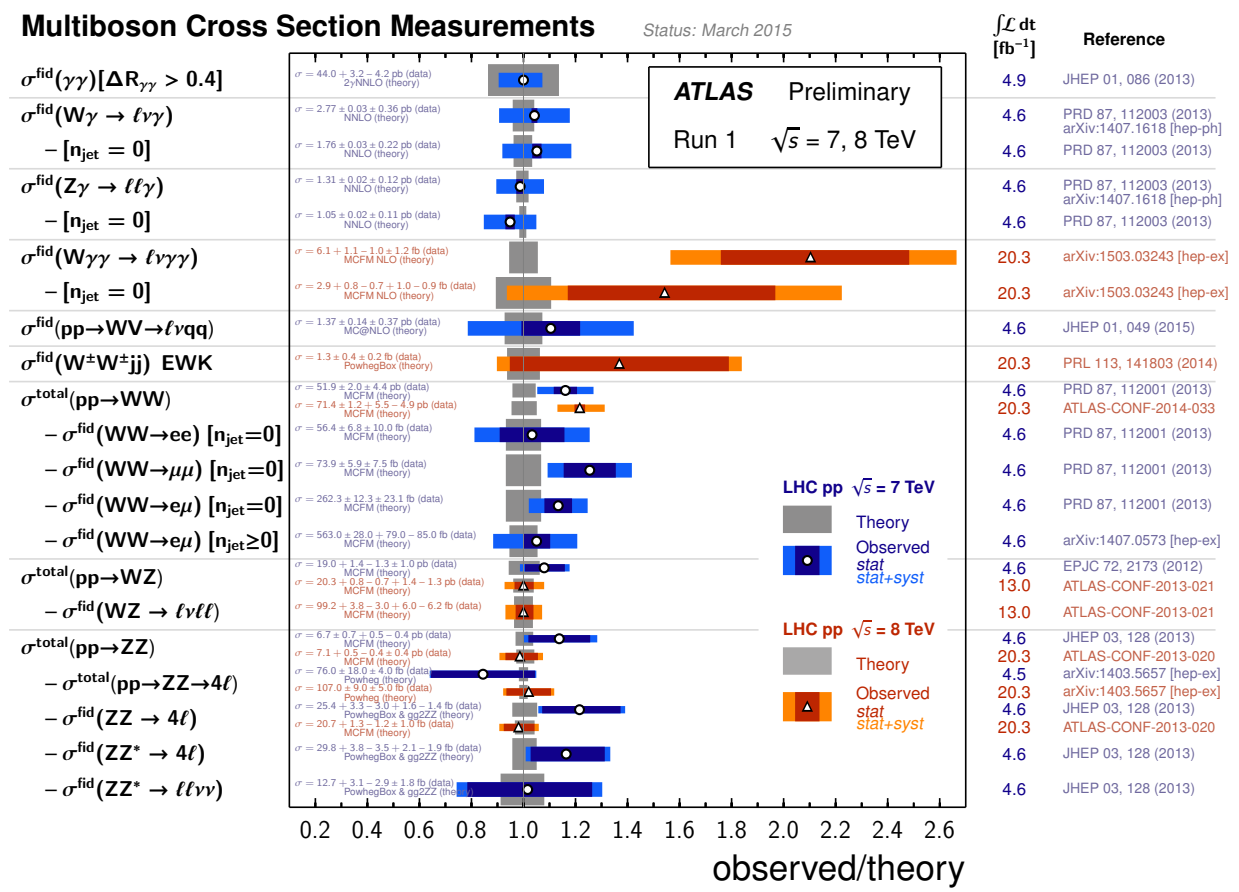

Fig. 8. The summary of ATLAS Multi-Boson production cross sections shown as the ratios of observed/theory. ${ }^{4}$ 
the measured ratio (observed/theory) and the uncorrected EPA (Equivalent Photon Approximation) calculations.

The BEC is measured by ATLAS using pairs of same-electric-charge charged particles with $p_{\mathrm{T}}>100 \mathrm{MeV}$ and $|\eta|<2.5$ from $p p$ collision data at $0.9 \mathrm{TeV}$ (7 $\left.\mu b^{-1}\right)$ and $7 \mathrm{TeV}\left(190 \mu b^{-1}\right.$ minimum-bias and $12.4 n b^{-1}$ high-multiplicity sample). ${ }^{20}$ Results of fitting the multiplicity $n_{c h}$, and the $k_{T}$ dependence of $R$ and $\lambda$ parameterized for BEC are shown in Table 1. Different functional forms and three different data samples are examined.

\section{Summary}

ATLAS performed a broad range of SM physics measurements covering a variety of SM physics aspects: QCD, Electroweak; V+jets, multi-boson, photon, jets physics. Most of the measurements are published with full dataset at $7 / 8 \mathrm{TeV}$. Measured cross-sections and parameterizations were well demonstrated and agree with SM theory predictions with high precision. A summary of the most recent ATLAS and CMS SM measured cross sections is shown in Figure 12 and 13. The results provide solid basis for new physics/phenomenon searches at ATLAS Run-I. The SM precise measurements will continue in ATLAS Run-II at a new center-of-mass energy of 13 $\mathrm{TeV}$ and consolidate the detector calibrations.

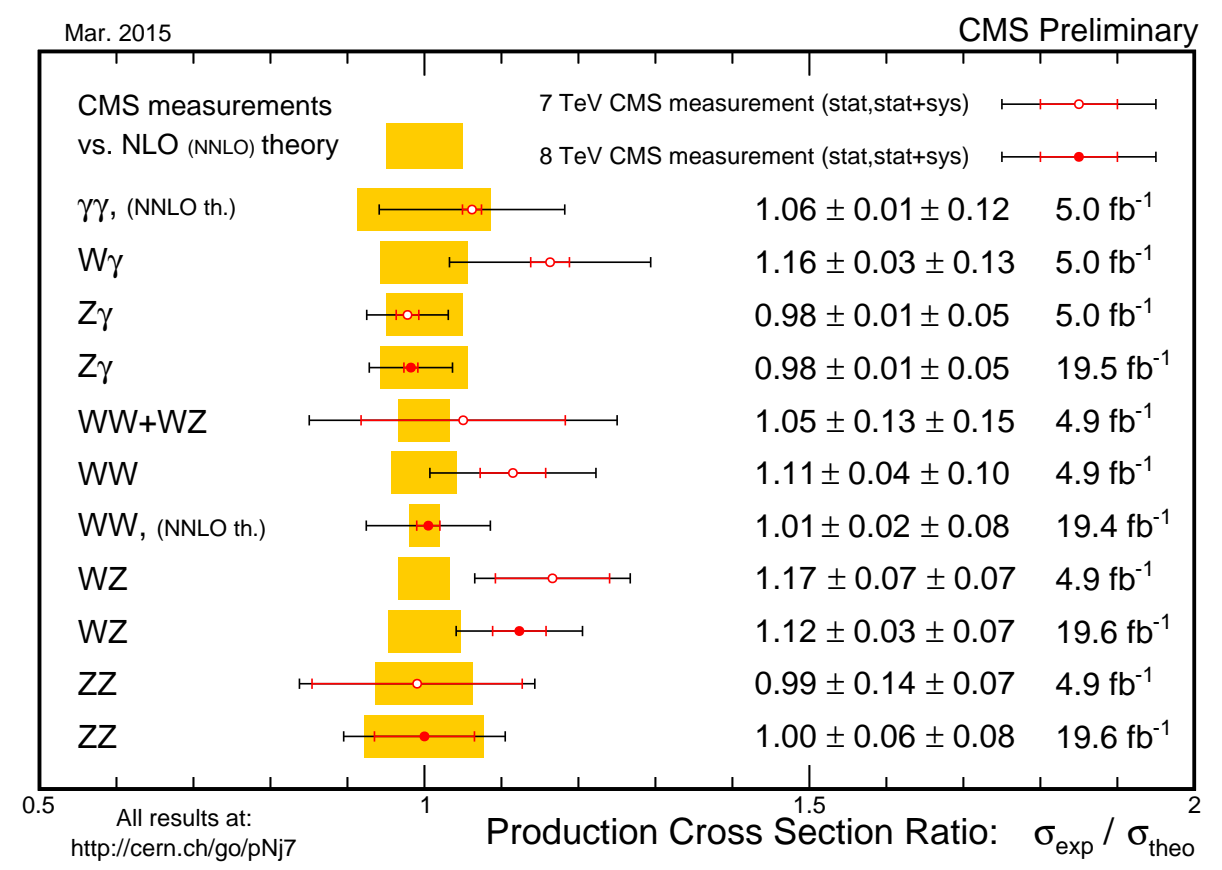

Fig. 9. The summary of CMS Multi-Boson production cross sections shown as the ratios of observed/theory. ${ }^{3}$ 


\section{References}

1. ATLAS Collab. JINST 3, S08003 (2008). ATLAS Collaboration, JINST 3 S08003 (2008).

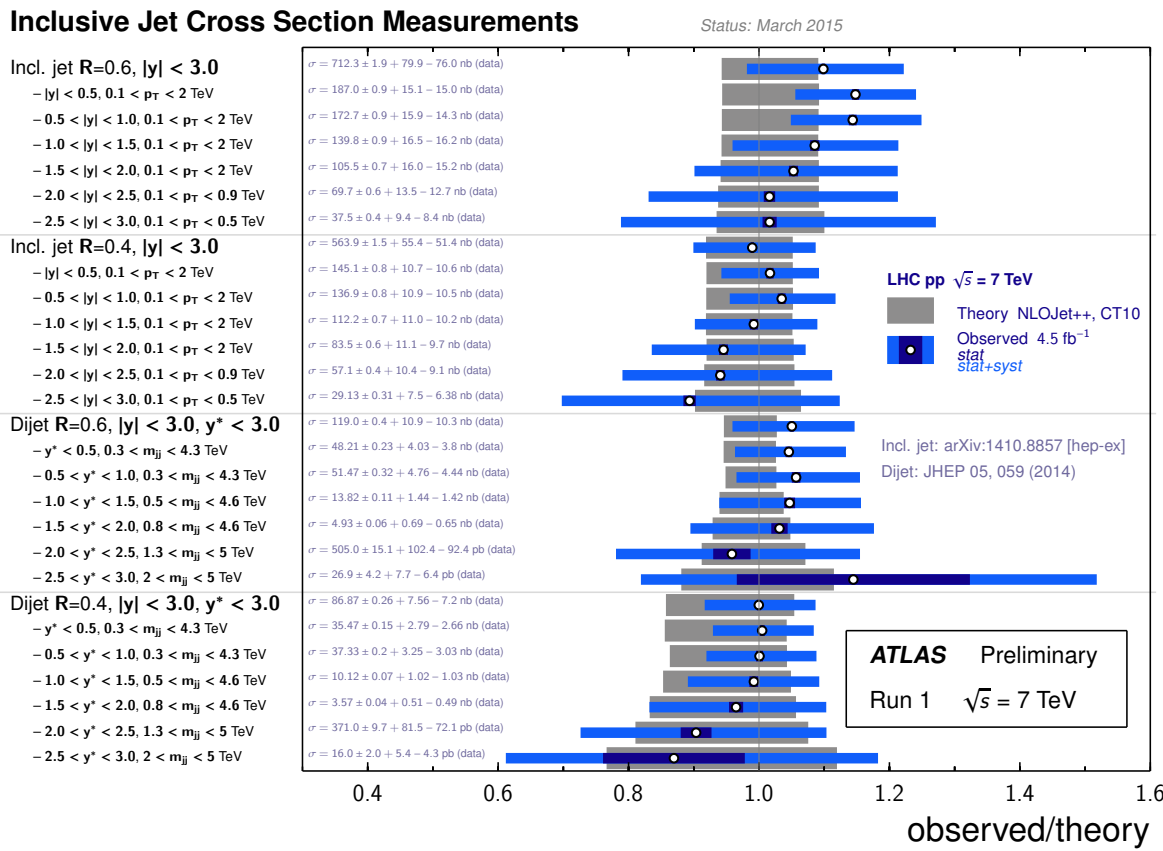

Fig. 10. Summary of the current ATLAS measured inclusive jet cross sections shown as ratio of observed/theory. ${ }^{18}$
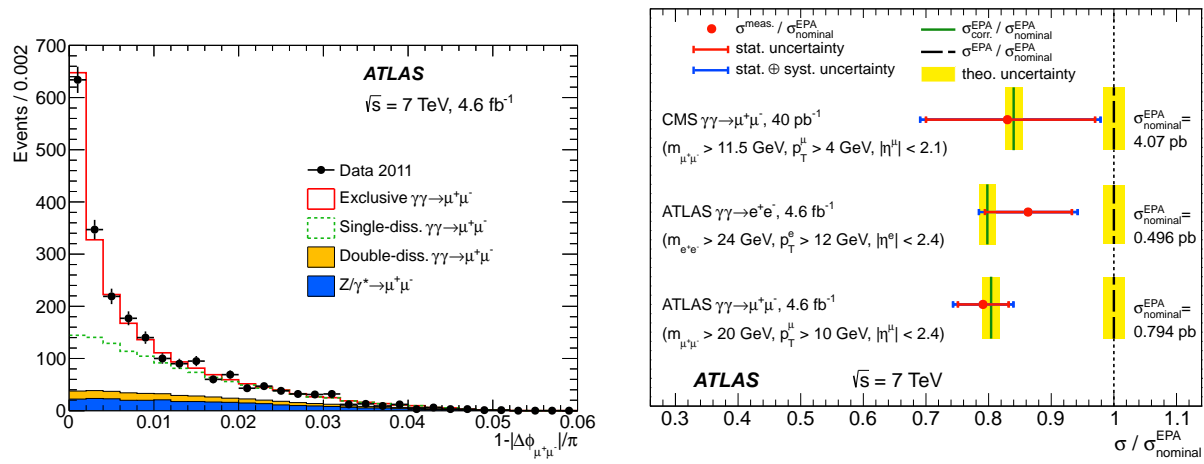

Fig. 11. Left: the acoplanarity distribution in $\mu \mu$ channel with exclusivity requirement. Right: the comparison between the measured ratio (observed/theory) and the uncorrected EPA calculations. ${ }^{19}$ 
2. CMS Collab. JINST 3, S08004 (2008).

3. CMS Collab. http://cms-results.web.cern.ch/cms-results/public-results/publications

4. ATLAS

Collab. https://twiki.cern.ch/twiki/bin/view/AtlasPublic/StandardModelPublicResults

5. ATLAS Collab. Eur. Phys. J. C 75, 82 (2015).

6. ATLAS Collab. Eur. Phys. J. C 74, 3168 (2014).

7. ATLAS Collab. J. HEP 09, 145 (2014).

8. ATLAS Collab. New J. Phys. 16, 113013 (2014).

9. ATLAS Collab. Phys. Rev. Lett. 113, 141803.

10. ATLAS Collab. Phys. Rev. Lett. 115, 031802 (2015).

11. ATLAS Collab. J. HEP 01, 049 (2015).

12. ATLAS Collab. Phys. Rev. Lett. 112, 231806 (2014).

13. ATLAS Collab., arXiv:1503.03709 [hep-ex], submitted to J. HEP.

14. ATLAS Collab. Phys. Rev. D 91, 052005 (2015).

15. ATLAS Collab. Phys. Rev. D 89, 052004 (2014).

16. ATLAS Collab. J. HEP 01, 086 (2013).

17. ATLAS Collab. Eur. Phys. J. C 75, (2015).

18. ATLAS Collab. J. HEP 02, 153 (2015).

19. ATLAS Collab. Phys. Lett. B 749, 242-261 (2015).

20. ATLAS Collab., arXiv:1502.07947 [hep-ex], submitted to Eur. Phys. J. C.

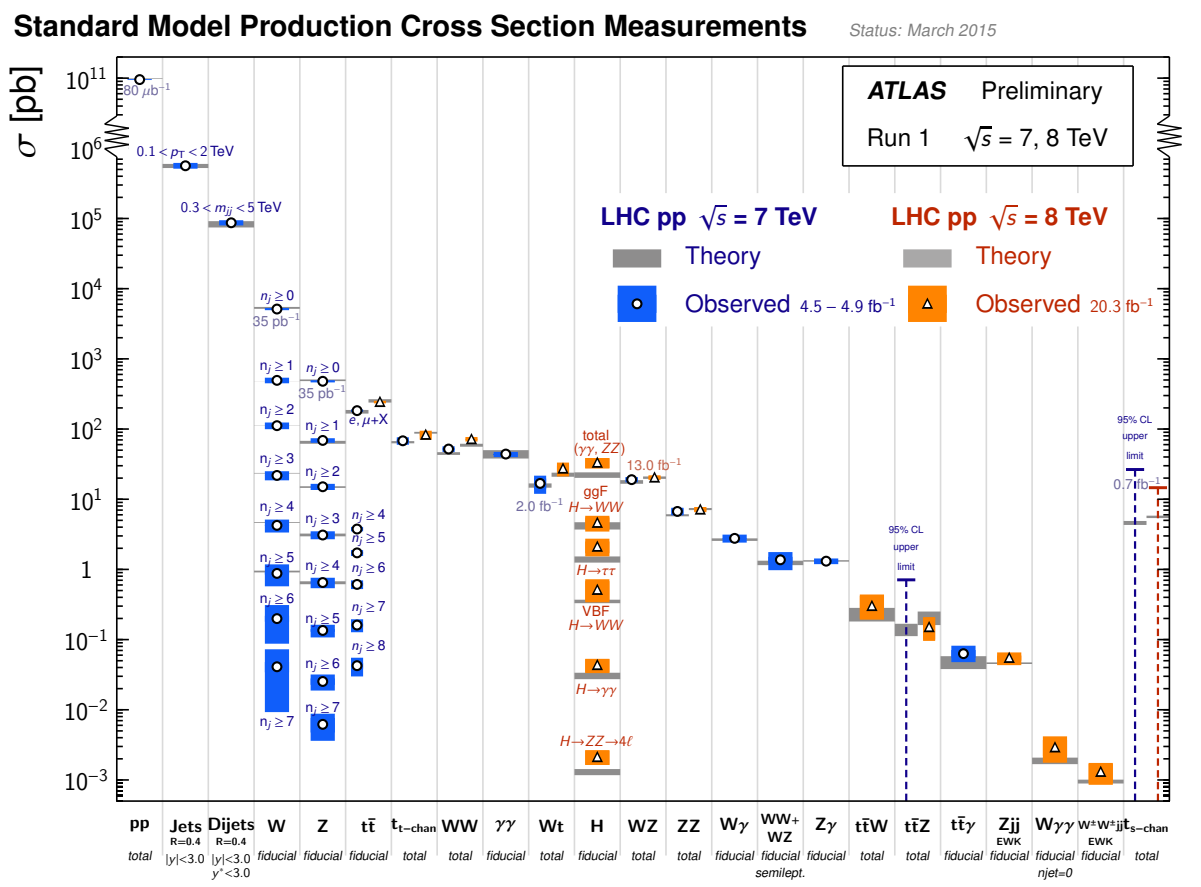

Fig. 12. Summary of the latest ATLAS SM total and fiducial cross section measurement results. ${ }^{4}$ 


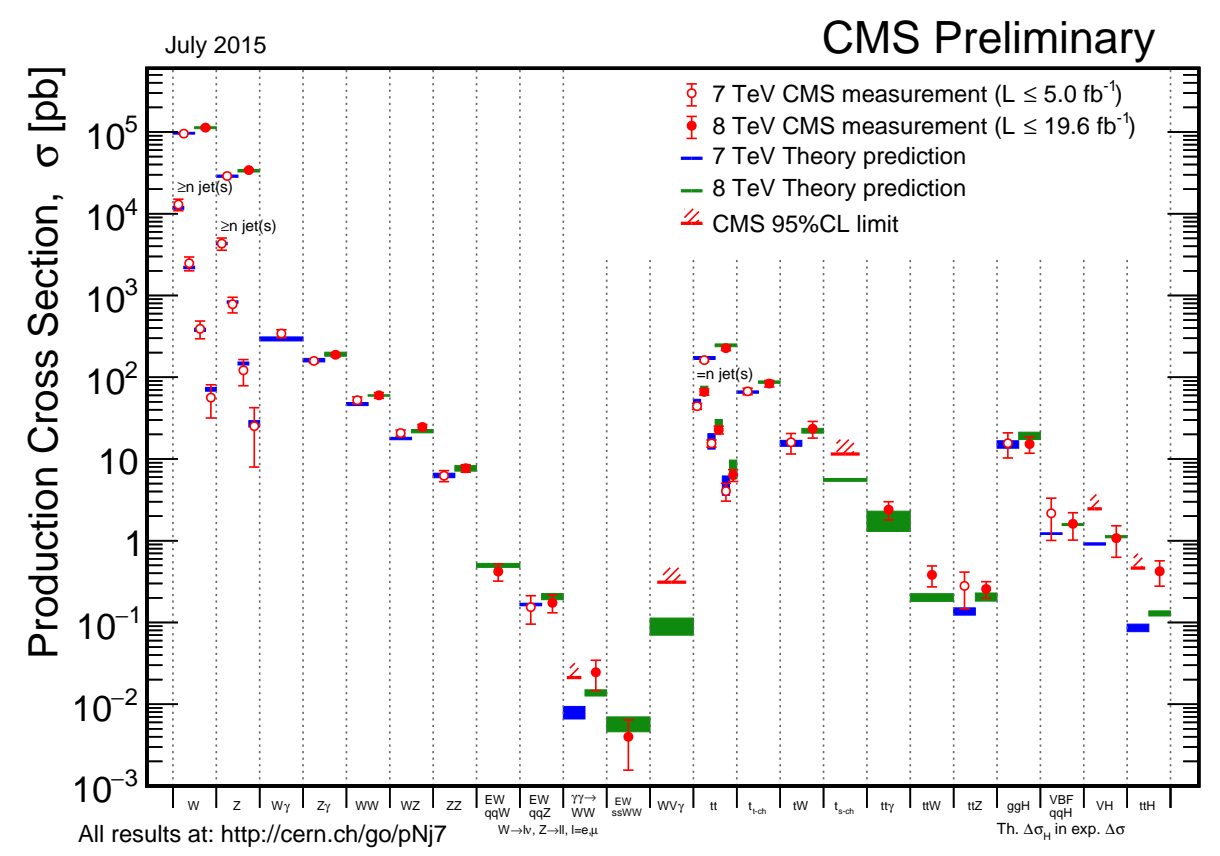

Fig. 13. Summary of the latest CMS SM total and fiducial cross section measurement results. ${ }^{4}$

Table 1. Results of fitting the multiplicity $n_{c h}$, and the $k_{T}$ dependence of $R$ and $\lambda{ }^{20}$

\begin{tabular}{|c|c|c|c|c|}
\hline \multirow{2}{*}{$\begin{array}{l}\text { BEC } \\
\text { param. }\end{array}$} & \multirow{2}{*}{$\begin{array}{c}\text { Fit } \\
\text { function }\end{array}$} & \multirow[t]{2}{*}{$0.9 \mathrm{TeV}$} & \multicolumn{2}{|c|}{$7 \mathrm{TeV}$} \\
\hline & & & Minimum-bias events & High-multiplicity events \\
\hline$R\left(n_{\mathrm{ch}}\right)$ & $\begin{array}{c}p_{0} \sqrt[3]{n_{\mathrm{ch}}} \\
p_{0}\end{array}$ & $p_{0}=0.64 \pm 0.07 \mathrm{fm}\left(n_{\mathrm{ch}} \leq 82\right)$ & $\begin{array}{r}p_{0}=0.63 \pm 0.05 \mathrm{fm}\left(n_{\mathrm{ch}}<55\right) \\
p_{0}=2.28 \pm 0.3\end{array}$ & $\mathrm{fm}\left(n_{\mathrm{ch}} \geq \overline{55)}\right.$ \\
\hline$\lambda\left(n_{\mathrm{ch}}\right)$ & $p_{0} \mathrm{e}^{-p_{1} n_{\mathrm{ch}}}$ & $\begin{array}{l}p_{0}=1.06 \pm 0.10 \\
p_{1}=0.011 \pm 0.004\end{array}$ & $\begin{array}{l}p_{0}=0.96 \pm 0 . \\
p_{1}=0.0038 \pm\end{array}$ & 0008 \\
\hline$R\left(k_{T}\right)$ & $p_{0} \mathrm{e}^{-p_{1} k_{T}}$ & $\begin{array}{l}p_{0}=2.64 \pm 0.33 \mathrm{fm} \\
p_{1}=1.48 \pm 0.67 \mathrm{GeV}^{-1}\end{array}$ & $\begin{array}{l}p_{0}=2.88 \pm 0.27 \mathrm{fm} \\
p_{1}=1.05 \pm 0.58 \mathrm{GeV}^{-1}\end{array}$ & $\begin{array}{l}p_{0}=3.39 \pm 0.54 \mathrm{fm} \\
p_{1}=0.92 \pm 0.73 \mathrm{GeV}^{-1}\end{array}$ \\
\hline$\lambda\left(k_{T}\right)$ & $p_{0} \mathrm{e}^{-p_{1} k_{T}}$ & $\begin{array}{l}p_{0}=1.20 \pm 0.18 \\
p_{1}=2.00 \pm 0.35 \mathrm{GeV}^{-1}\end{array}$ & $\begin{array}{l}p_{0}=1.12 \pm 0.10 \\
p_{1}=1.54 \pm 0.26 \mathrm{GeV}^{-1}\end{array}$ & $\begin{array}{l}p_{0}=0.75 \pm 0.10 \\
p_{1}=0.91 \pm 0.45 \mathrm{GeV}^{-1}\end{array}$ \\
\hline
\end{tabular}

